\title{
UNA PROPUESTA DE TRABAJO DESDE LA EVALUACIÓN INTERDISCIPLINARIA DE UNA NIỸ̃A CON DISCAPACIDAD: IMPLICACIONES PARA UNA PRÁCTICA CLÍNICA
}

\author{
Rosa Figueroa Sánchez ${ }^{1 .}$ \\ Sylvia Martínez Mejías ${ }^{2}$
}

...las ciencias progresan por los bordes y éstos contactan con otras ciencias. Las interfases asi generadas constituyen áreas muy fructiferas para el progreso del conocimiento, y éste sólo puede lograrse con el trabajo interdisciplinario (Lejarraga, 2004).

\section{Resumen}

$\mathbf{E}$ ste artículo examina la evaluación interdisciplinaria de una niña con discapacidad. Se presenta una mirada crítica a los factores fragmentados de la evaluación tradicional y por otro lado la importancia de integrar nociones ideas y conceptos entre las disciplinas, específicamente psicología y trabajo social. El trabajo interdisciplinario permitió una mirada amplia al desarrollo integral de la niña y su contexto social.

Descriptores: evaluación interdisciplinaria, discapacidad, intervenciones clínicas, familia.

\footnotetext{
1 Instructora en la Escuela Graduada de Trabajo Social Beatriz Lassalle y Trabajadora Social en el Centro Pediátrico de Servicios Habilitativos, Hospital Pediátrico Universitario, Centro Médico, San Juan, PR.

${ }^{2}$ Catedrática Auxiliar en el Departamento de Psicología y Psicóloga Clínica en la Clínica de Servicios Psicológicos, San Juan, PR.
} 


\section{Abstract}

This article presents an interdisciplinary approach to the assessment of a girl with disability. The evaluators critically looked at the fragmented factors of standard evaluation procedures, while on the other hand emphasized the importance of integrating notions, ideas, and concepts among disciplines, specifically Psychology and Social Work. The interdisciplinary work allowed for a broad look at the integral development of a girl and her social context.

Key words: interdisciplinary evaluation, disability, clinical intervention, family.

\section{Introducción}

Este trabajo surgió entre cuestionamientos y debates sobre acercamientos teóricos a la discapacidad. Se planteó la idea de desarrollar trabajo interdisciplinario en esta área dado la complejidad de la misma. Es evidente que la naturaleza del trabajo requiere que los sujetos que asumen un saber dentro de una disciplina en particular, sean capaces de traspasar el mismo, reconociendo por una parte sus limitaciones, y por otra, las aportaciones de disciplinas relacionadas. Trabajo arduo y que sólo se produce en la marcha. Es desde ahí que se sentaron las bases para el trabajo que se ha de elaborar.

Cuando se piensa en trabajo en equipo se parte de la premisa de que muchas personas comparten la tarea. Sin embargo, la idea que elaboramos a la luz de un caso clínico fue pensar el dolor humano, el integrar nociones y desarrollar intervenciones desde una mirada en común, lo que trasciende el trabajo en equipo tradicionalmente concebido. Entendiéndose por mirada en común un posicionamiento teórico desde un marco analítico. El tema de la discapacidad es sumamente complejo. Por lo tanto no puede circunscribirse a la particularidad de disciplina alguna, sino que exige una mirada abarcadora y un trabajo interdisciplinario para su estudio y abordaje (Dell'Anno, 2004). La naturaleza de este acercamiento exige que aportemos conceptual y metodológicamente nuestro quehacer en las 
disciplinas; reconociendo que existe una confluencia teórica al apalabrar los distintos aspectos de la problemática.

\section{Algunas conceptuaciones}

Los conceptos multidisciplinario, interdisciplinario y transdisciplinario han sido muy debatidos, particularmente en la clínica infantil (Amores, 2000; Fainblum, 2004; Linder; 2008). De acuerdo a Fainblum (2004), "lo multidisciplinario se remite a una multitud de disciplinas, encerradas en sí mismas, dándose una yuxtaposición de discursos, desarrollándose en forma aislada una de otra, con tratamientos múltiples y simultáneos"(p. 102). Bajo esta definición las disciplinas pueden o no relacionarse entre sí. En la evaluación tradicional hay una tendencia a elaborar informes sobre la ejecución o el funcionamiento de un sujeto para opinar sobre el mismo. Esta dinámica se lleva a cabo sin que la opinión de otro profesional sea parte del informe redactado o de las recomendaciones emitidas. Son saberes independientes que intentan dar cuenta del funcionamiento de un sujeto desde una disciplina, partiendo de la premisa que cada discurso es certero. No sólo se emite una opinión pericial desde una disciplina sino que se olvida considerar el saber que posee el propio sujeto en cuestión.

Fainblum (2004) también define lo interdisciplinario, como aquello que: "remite al concepto de estructura, a una determinada relación entre las disciplinas donde cada una, al ser un término de la estructura, presentan entre sí una interdependencia" (p.102). En esta concepción existe un discurso en relación a otros. Las disciplinas se entrecruzan facilitando un espacio discursivo sobre el cual mirar el dolor humano, donde el sujeto no es visto desde un lugar en las disciplinas, sino desde una frontera. De tal manera no es un discurso que se asigna en forma de diagnóstico arbitrario sino que se construye en función de la queja que se presenta. Para esta autora lo transdisciplinario es "una posición, en tanto se trata de un atravesamiento común de las distintas disciplinas, no por una teoría hegemónica, sino por una concepción ética que resitúa en un posicionamiento común las diferentes prácticas y por lo tanto las intervenciones" (Fainblum, 2004:102). Es desde la mirada común que 
podemos dar cuenta de la queja humana sin fragmentarla a la luz del saber de las disciplinas en cuestión, psicología y trabajo social. El asumir esta postura implica una posición metodológica alterna a las planteadas por dichas disciplinas. Se trata de crear un espacio de trabajo común en el que se trascienda la disciplina por sí misma dando lugar a que el saber no se imponga sino que se construya.

El proceso de evaluación que presentamos en este trabajo se abordó desde una posición interdisciplinaria. Hubo una puesta en común de una postura analítica y un marco referencial. Esto fue posible dado a que las disciplinas en juego, trabajo social y psicología, se entremezclaron asumiendo un mismo marco referencial. Fainblum (2004) plantea además, que cada disciplina debe estar firme en su conocimiento pero a la vez flexible para abrir espacio a nuevos saberes. Es a partir del impacto que tuvo el proceso evaluativo en la familia y en las autoras, lo que llevó a plantear la importancia de crear un espacio discursivo en el que no sólo el ejercicio teórico se pueda plasmar sino también el práctico. Es vital desarrollar espacios físicos de práctica conjunta así como concienciar a los profesionales para traspasar la segmentación del conocimiento o el saber.

La función diagnóstica tradicional está delimitada por el psicólogo o el psiquiatra. Esto es una posición histórica que han tenido los sistemas de salud en el país. Lo anterior influye, entre otros, en la mirada y significación que tiene un profesional ante la discapacidad. Al examinar esta posición observamos que se ha dado una significación del diagnóstico hacia explicaciones de orden biológico perpetuando la dicotomía entre cuerpo y psiquis. Ese proceso excluye considerar la significación de la familia ante su dolor y la dimensión social de la condición. El uso y costumbre de la práctica de la evaluación en Puerto Rico perpetúa el relacionar el diagnóstico con la evaluación estructurada o sistemas de clasificación normalizados (Bernal \& Rodríguez, 1992). Para esto, en ocasiones, sólo se utilizan instrumentos de medición, eliminando la posibilidad de significación a través de métodos tan válidos como por ejemplo, el análisis de sesiones de juego. Romper con lo tradicionalmente aceptado crea resistencia entre los profesionales, perjudicando en última instancia al sujeto. Considerar métodos no estandarizados pero de gran validez 
teórica como la observación clínica, visitas al hogar, trabajo en salón de juego, permite girar la mirada hacia el sujeto y no el sujeto hacia el instrumento.

Serrat (2204) plantea que se debe reconocer que toda clasificación es arbitraria en el sentido de que las condiciones allí estipuladas responden a una particular valoración sobre lo que se desea ordenar. Sin embargo, el hecho de ser arbitraria no equivale a decir que sea caprichosa, irrazonable o injusta, sino que es el producto de una investigación y selección de posibles interpretaciones (p.64).

Es precisamente un trabajo de investigación el que se genera cuando dos o más profesionales de la salud entrecruzan los saberes en un espacio común y debaten las ideas sobre el tema en cuestión. Entre la psicología y el trabajo social convergen conceptos e ideas que abordan la complejidad humana reconociendo que está constituida por procesos multifảctoriales con relaciones intensas entre sí. La experiencia como evaluadoras clínicas nos ha permitido reconocer que el enfoque multidisciplinario en Puerto Rico es habitualmente utilizado. Siendo este enfoque en ocasiones uno disociativo y de mirada fragmentada. Dell'Anno (2004) expone que las miradas fragmentarias pueden ser un accionar irreflexivo que responde a la petición de respuestas rápidas, a necesidades institucionales y a políticas públicas, teniendo una repercusión nefasta en el sujeto y sus familias.

Por una parte, el enfoque fragmentado no facilita el que las familias ordenen el significado de la discapacidad. Esto repercute en un juego entre el saber y no saber. Se genera conocimiento, sin embargo, no se integra ese saber en función del sujeto y su familia. El conocimiento pasa a ser entonces meras palabras que describen, estableciendo entre ellas y el sujeto una distancia. En cierto modo esta distancia sirve como protección para no saber. Las miradas fragmentadas fungen como miradas autísticas en las que un diagnóstico o la descripción de síntomas se emiten sin la posibilidad de que el sujeto y sus familias las apropien y las signifiquen. 
La mirada fragmentada de los profesionales no permite que ese saber, por doloroso que sea, cobre significado en el núcleo familiar, lo cual obstaculiza que la familia se construya y reconstruya en función del sujeto y su discapacidad. De igual modo, esta fragmentación no permite que el estado asuma la responsabilidad social de cumplir con los derechos humanos de las familias al desarrollar un programa de habilitación con los servicios especializados que ello requiere. Por último, la demanda de respuestas inmediatas, que se reduce a demandas costo-efectivas, libera al estado de responder a la inversión económica que requiere el sujeto con discapacidad y su familia.

\section{Descripción de la metodología}

Este trabajo fue realizado en el mejor interés de profundizar en el significado que tiene la discapacidad de una niña para los miembros de su familia. Identificamos los factores protectores de ésta familia para realizar las tareas diagnósticas, entendiendo que es la familia quien define el proceso a seguir en las intervenciones. Reconocemos la evaluación clínica como un proceso interaccional y dialógico a través de un continuo de observaciones que varía de un nivel de análisis individual a uno de la díada y del aspecto familiar. A su vez la evaluación requiere un procedimiento coherente, ordenado y detallado como producto de un enfoque clínico.

Las evaluadoras accionan este procedimiento en tanto realizan conjuntamente el proceso de evaluación, integrando a la familia en el mismo. Posteriormente, discuten los hallazgos y generan sólo un informe de evaluación. Sin embargo, a pesar de la integración de las disciplinas, el contar sólo con algunos instrumentos en común y utilizar lo que cada disciplina aporta, hace que el espacio de trabajo sea considerado como uno interdisciplinario.

Nuestra orientación metodológica es el estudio de caso. Desde esta perspectiva intentamos relacionar y significar la información que la práctica nos ha brindado a partir de una posición psicodinámica. Su carácter es descriptivo y tiene una única unidad muestral. 


\section{Proceso de evaluación interdisciplinaria}

\section{Entrevista familiar}

Iniciamos el proceso de evaluación mediante una reunión con la niña y sus progenitores. Esto permitió la observación de la interacción familiar. Identificamos y validamos las necesidades de la familia para en función de éstas desarrollar el proceso de evaluación así como las intervenciones. Fue necesario reconocer los aspectos importantes de la dinámica familiar, de las pautas de crianza y la relación vincular. En la evaluación de la relación vincular observamos particularmente la calidad de ésta. Durante el proceso de entrevista familiar se evaluaron seis áreas específicas: el vínculo prenatal que la madre y el padre establecieron con la niña; la historia familiar tanto del padre como de la madre y la significación que éstos dan a la misma; la calidad del apego y la disposición emocional de ambos progenitores para reconocer las necesidades de la niña; el apoyo que se dan en el proceso de crianza; la capacidad de la familia para expresar variedad de afectos; y la observación de los patrones de comunicación entre los miembros de la familia.

\section{Observación del juego familiar}

Esta es una situación de juego planificado en la que se provee a los progenitores el material de juego y se analizan sus estilos de interacción con la niña (Gil, 1994). En las actividades que ellos seleccionaron observamos la interacción social que los involucra, el placer que experimentan al jugar, y las modificaciones que el padre o la madre realizaron para que la niña participara de la actividad.

\section{Observación del juego de la niña}

Esta es una observación de juego libre en el que la niña elige el material de juego. Se examinó el uso del material seleccionado por la niña y la pertinencia para su nivel de desarrollo y habilidades. Además, se valoró la calidad de la relación con las evaluadoras. Las observaciones de juego libre se realizaron en dos sesiones; en una de ellas los progenitores no estaban en el salón. El objetivo fue analizar el proceso de separación entre la niña y sus progenitores. 


\section{Visita al hogar}

Esta constituye una observación de la interacción social de la niña y su familia en su ambiente natural. Se realizó una evaluación del espacio físico para maximizar y habilitar el espacio en función de las necesidades familiares (Mclean, Bailey \& Wolery, 2003). La visita se llevó a cabo durante el proceso de alimentación y juego. La literatura resalta la importancia de evaluar durante periodos en el que se cubren necesidades básicas.

\section{Visita al preescolar y la escuela}

Se observó el manejo del espacio, sus interacciones con pares y figuras de autoridad así como su proceso de formación académica en etapas escolares. Se evaluó la adaptabilidad del espacio a las necesidades de la niña (Mclean, Bailey \& Wolery, 2003).

\section{Evaluación estructurada}

Se administraron una serie de pruebas psicológicas para obtener un perfil del funcionamiento de la niña. En esta parte del proceso de evaluación una de las evaluadoras junto a los progenitores, permanecieron observando a través de un espejo unidireccional. Para obtener un índice de habilidades cognitivas'se administró la Escala de Ejecución Internacional Leiter-Revisada, por ser un instrumento sensible a las necesidades en el lenguaje. Se utilizó la Prueba de Integración Visual-Motora Bender Gestalt que evalúa destrezas grafomotoras. Por su parte, la Prueba de Aprovechamiento-Revisada Woodcock Muñoz proveyó un perfil de habilidades académicas. Se envió a la escuela el Inventario de Comportamiento Escuela (IDC-E). El instrumento es una escala que recoge las observaciones de maestros y maestras en el salón de clases. Se administró además, dos escalas de autismo (CARS y GARS) con el propósito de obtener indicadores de dicha condición debido a las quejas presentadas por la familia. Los instrumentos proveyeron un perfil de las habilidades de la niña en diversas áreas. Posteriormente se analizaron los datos en función de su contexto social y familiar. 


\section{Observaciones del proceso de evaluación}

\section{Presentación de caso}

E. es la única hija del matrimonio. El embarazo para E. fue complicado. Su madre tuvo síntomas de depresión durante el embarazo. Su desarrollo psicomotor fue adecuado. Con relación al desarrollo del lenguaje se informó que E. presentó rezagos significativos. Su conducta evidenció dificultades en la interacción social. Los progenitores fueron receptivos a las necesidades de la niña aunque manifestaron angustia ante el desconocimiento del manejo de las mismas.

\section{Primer proceso de evaluación}

Durante el primer proceso de evaluación, E. tenía dos años con siete meses. La familia mostró un vínculo parental fuerte hacia la niña, con poca reciprocidad por parte de ésta. Ante esa situación la madre particularmente expresó un gran sentimiento de culpa por las dificultades de la niña. Esto ligado a los sentimientos de rechazo inicial al proceso de gestación. Por otro lado, el padre se mostró comprensivo ante las necesidades tanto de la madre como de la niña. A pesar del rechazo de la niña, el padre continuamente estableció los acercamientos afectivos. En las instancias en que la niña mostró reciprocidad afectiva, manifestó mayor agrado con la interacción del padre en comparación con la de la madre. Las evaluadoras trabajaron con esas observaciones facilitando la significación de las conductas, reconociendo el discurso dominante sobre la maternidad.

\section{Proceso de reevaluación}

La familia solicitó una reevaluación luego de cuatro años para revisar el diagnóstico de su hija y conocer las fortalezas en el desarrollo psicosocial. La familia había confrontado serias dificultades con los servicios recibidos a través del sistema de educación. Existía una pugna entre lo que el sistema consideraba apropiado para la niña y lo que la familia entendía era la mejor ubicación de acuerdo a las necesidades de su hija. E. tenía seis años con seis meses. Durante los años intermedios entre ambas evaluaciones la familia contó con 
apoyo profesional para consultas con las evaluadoras sobre el desarrollo de su hija.

En la reevaluación se observó que el matrimonio elaboró de forma clara y organizada las preocupaciones sobre su hija. Lograron acuerdos sobre la crianza de la niña. Estos mantuvieron las reglas y los límites de manera consistente ante la conducta de E. Por su parte, la niña fue inconsistente al responder a dichas reglas. Esta demandó mucha atención, y mostró persistencia en sus pedidos. Los progenitores utilizaron estrategias adecuadas para lidiar con la conducta de su hija. Estos reconocieron y anticiparon las necesidades e intereses de la niña ofreciendo respuestas oportunas. Se mostraron cálidos, amables y sensibles con E. Se observó humor, cercanía y tolerancia como una manera de resolver los conflictos y mantener una atmósfera emocional adecuada. E. mostró reciprocidad a las expresiones de afecto que sus progenitores le brindaron. Se observó un vínculo emocionalmente íntimo y significativo entre ellos. Además reflejaron una actitud receptiva para comprender las necesidades en el desarrollo presentadas por la niña.

Durante la segunda evaluación los progenitores supieron elegir los materiales de juego de acuerdo al nivel de dificultad de su hija. Se observó que escogieron los materiales con los que la niña logró atención sostenida y eliminaron aquellos con los que se podía desorganizar. Lograron una atmósfera de juego placentera, facilitando que la niña se involucrara en el juego interactivo.

En el proceso de juego con las evaluadoras E. mostró reciprocidad social. La niña respondió a preguntas sencillas pero no inició conversación alguna. Sus interacciones fueron mayormente no verbales, sin embargo, habló en frases de forma espontánea. Su habla fue peculiar, dando la impresión de que estaba cantando. La niña hizo ruidos rítmicos con la boca en actividades pasivas, aparentemente auto estimulándose. E. estableció contacto visual, sin embargo no lo mantuvo de forma espontánea. Utilizó el material de juego con propósito y variadamente. E. mostró juego imaginario y exploró con confianza el ambiente. Logró mantenerse en una actividad por tiempo prolongado. Durante una segunda sesión la niña mostró un 
nivel de actividad moderadamente alto y tuvo dificultad para la atención sostenida con el material de juego. E. tendió a quitarse los zapatos y a correr por el salón de evaluación. La separación de sus progenitores fue apropiada.

En el salón de clases E. se distrajo jugando con su lápiz, su camisa y sus manos. No logró seguir la instrucción general que se le ofreció al grupo. La asistente le tuvo que dar la instrucción de forma individualizada y la niña respondió apropiadamente bajo ese estilo. Tendió a hablar sola sin seguir la misma temática presentada en clase. Ante una solicitud de su maestra, la niña mantuvo silencio y sólo respondió cuando la asistente la dirigió a la tarea. E. no interactuó con sus pares por iniciativa, sin embargo, aceptó sin dificultad el acercamiento de éstos. Su trabajo escolar reflejó que domina responder a preguntas sobre hechos concretos pero no logra hablar sobre detalles y características específicas. La niña no logró entender situaciones de moraleja ni interpretó contextos de humor. En ocasiones ofreció respuestas inapropiadas a preguntas de un cuento específico trabajado en el salón. Mencionó hechos previos discutidos en el salón fuera de contexto. E. reconoció cuando una tarea le era compleja y señaló en varias ocasiones "me fallé". A pesar de identificar tareas difíciles, continuó con las mismas sin cambiar su estado de ánimo. En ocasiones dio la impresión de que el fracaso le era indiferente.

En el comedor E. siguió la rutina al servírsele los alimentos. Se sentó sola a pesar de tener otras niñas a su alrededor. La niña usó apropiadamente la cuchara pero al cabo de un rato, jugó con la misma lamiéndola como si fuera una paleta. Posteriormente, la asistente la redirige a la tarea diciéndole "E. come", y la niña continuó con su proceso de alimentación. En dos ocasiones subsiguientes asumió una actitud inexpresiva, mirada perdida, y dejó de comer. Nuevamente la asistente la redirige a la actividad con el mismo comentario: "E. come". Al terminar sus alimentos llevó la bandeja al área de desperdicios en donde consistentemente una empleada le dirige el proceso. E. interrumpió la actividad debido a que la empleada del comedor se movió del mostrador donde acostumbra estar. La niña 
abandonó la bandeja y la asistente nuevamente la redirigió a la tarea de limpiar la bandeja.

Durante el recreo E. corrió de un lado para otro, principalmente siguiendo a otras niñas. E. identificó a sus compañeras como amigas pero no utilizó los nombres propios para llamarlas. No inició interacción con pares ni rechazó sus acercamientos. E. no conversó con éstas sino que las niñas se dirigieron a ella. E. se refirió a sí misma en tercera persona.

\section{Experiencias con el sistema educativo}

A los dos años aproximadamente la niña ingresó a un centro preescolar. E. permaneció en el centro alrededor de cinco meses. A los tres años asistió a un centro especializado para niñas con discapacidad, en el que logró adaptarse rápidamente. El programa era organizado lo que le proveyó rutina y seguridad. El personal logró identificar las fortalezas y necesidades de E. utilizándolas en la estimulación temprana. Esto facilitó el progreso productivo de la niña. E. pasó a cursar kinder en una escuela de la comunidad. En la misma se le ofreció asiento preferencial y apoyo individualizado. Se observó progreso significativo en su lenguaje expresivo comenzándolo a utilizar en su interacción con pares. En este plantel E. tuvo buen rendimiento académico. Durante el primer semestre de primer grado, los progenitores cambiaron a la niña de escuela debido a que no tenía maestra asignada. La matricularon en una institución privada. Sin embargo, el plantel no estaba preparado para atender las necesidades de E. y pidieron a los progenitores buscar otras alternativas académicas. Posteriormente la ubicaron en otra escuela de comunidad. En ese plantel la maestra se ausentó de forma significativa por lo que los progenitores hicieron una querella al Departamento de Educación. Finalmente la reubicaron en otra escuela de la comunidad. Al momento de la evaluación la niña participaba de un programa de corriente regular asistida por un Trabajador $\mathrm{I}^{3}$ y

\footnotetext{
${ }^{3}$ En el Sistema de Educación de Puerto Rico tienen la función de asistir al niño o niña en sus necesidades tanto físicas como educativas para que puedan completar las tareas asignadas en el ambiente escolar.
} 
recibía clases bajo el Programa de Educación Especial en un salón recurso.

Sobre la evaluación estructurada los resultados evidenciaron que E. tiene un funcionamiento cognoscitivo a nivel promedio, cuando se compara con niños y niñas estadounidenses ${ }^{4}$. Los resultados indicaron que los rezagos en el lenguaje no han interferido en la adquisición de destrezas cognoscitivas. Sin embargo, de los resultados se infirió que E. podría realizar mejor trabajo académico si recibe asistencia directa. Tiene mucha dificultad para reconocer y analizar claves sociales por lo que carece de las destrezas necesarias para analizar situaciones que se dan cotidianamente. Esto último a su vez interfiere con el desarrollo de destrezas interpersonales.

Con relación a las destrezas escolares los resultados reflejaron un funcionamiento a nivel promedio en el área de lectura simple y bajo el promedio en la comprensión de lectura. En el área de cálculo obtuvo un funcionamiento a nivel superior, pero en el área de problemas aplicados reflejó un funcionamiento muy bajo. Los resultados son consistentes con las necesidades evidenciadas por la niña. E. tiene gran dificultad en la aplicación de conceptos. Por otra parte, en dictado obtuvo una puntuación a nivel promedio y bajo promedio en el área de redacción. La niña tiene dificultad en la construcción de oraciones. Esos resultados no son consistentes con su potencial intelectual. Lo anterior sugiere que las dificultades para atender y su desorganización están interfiriendo con el rendimiento de E.

\section{Relevancia de un trabajo interdisciplinario}

La evaluación interdisciplinaria se constituye como un instrumento significativo a partir de la experiencia de problematizar y reflexionar desde la perspectiva de las personas involucradas. Por lo que la redacción de un informe de evaluación interdisciplinaria no puede elaborarse desde un contexto utilitario, dando cuenta exclusivamente

\footnotetext{
${ }^{4}$ La prueba utilizada no cuenta con normas para la niñez puertorriqueña, por lo que se compara con las normas desarrolladas por el instrumento. En Puerto Rico no contamos con pruebas no verbales normalizadas para nuestra población.
} 
sobre el retraso en el desarrollo y ofreciendo recomendaciones para contribuir al progreso. Por su parte, el trabajo clínico que se realizó reconoce que cada uno de los sujetos involucrados tiene una manera particular de mirar la realidad, en este caso el desarrollo psicosocial de E., y que permite encontrar relaciones, acuerdos y confrontaciones entre las distintas personas. Un análisis cualitativo de las habilidades de la niña permitió entender sus fortalezas y necesidades más allá de su funcionamiento con su grupo de pares.

El informe de evaluación interdisciplinario se enfocó en una posición interaccional. De tal manera que no se hizo énfasis en la definición de un problema y sus recomendaciones, sino en las dinámicas y los procesos, lo que permitió que la familia identificara sus fortalezas y determinara el curso de acción a seguir. La familia participó durante el proceso de evaluación como miembros del equipo, conjuntamente con ellos se eligieron los criterios e instrumentos evaluativos. Estos se mantuvieron físicamente en el espacio que se utilizó para realizar la evaluación interdisciplinaria. En términos metodológicos los progenitores de E. no estuvieron en la observación que se realizó en el ambiente escolar. En la práctica cotidiana, los progenitores no se encuentran en el salón mientras se desarrolla la clase. Sin embargo, se llevó a cabo una sesión en la que los progenitores revisaron las libretas y otros materiales escolares de E., y elaboraron sus observaciones sobre el aprovechamiento de la niña.

En la redacción del informe valoramos cualitativamente el vínculo afectivo entre la niña y su familia. Se informó sobre la conducta adaptativa de E. y se consideraron los patrones de comportamiento que la familia había observado. Se analizó el nivel de acuerdo entre los diferentes instrumentos utilizados y relacionamos entre sí toda la información disponible. Los resultados del proceso se informaron de forma clara y sencilla permitiendo que la familia trabajara con la información en vez de ser meros receptores del proceso. La familia logró trabajar con la información al hacer uso de preguntas abiertas que les permitió resituar e interpretar el desarrollo psicosocial de su hija. 
La naturaleza del trabajo descrito fue posible sólo mediante un acercamiento interdisciplinario en tanto este ofrece una visión integral de los sistemas complejos. Como señala Lejarraga y Schejter (2004), la interdisciplina posibilita el surgimiento de nuevas preguntas y permite respetar la complejidad del sujeto y así evitar el riesgo de la fragmentación desde la mirada especializada. Estas nuevas preguntas se abren a partir de la palabra de los progenitores que interpelan a las evaluadoras a no mirar a la niña en su déficit y que sus conductas tienen un significado construido en el contexto o interacción social.

Una de las aportaciones de la evaluación interdisciplinaria es su validez terapéutica. Esto se constató a través del análisis de los tiempos del proceso evaluativo. Durante el primer y segundo proceso de evaluación ocurrió un intervalo de cuatro años con tres consultas por parte de los progenitores a las profesionales. En estas consultas articularon su queja no como demanda sino como parte de un proceso de análisis que se dio en conversación de un progenitor al otro. Ante esto Dolto (1994b) señala que cuando la madre y el padre reflexionan con calma y honestamente, sintiéndose escuchados, comprenden que ellos poseen los medios para resolver sus problemas.

\section{Conclusión}

Las divergencias entre profesionales se pueden adjudicar a las diferencias paradigmáticas. Se recomienda entonces una integración colaborativa y un trabajo más cercano, en el que cada miembro pueda adquirir conocimientos sobre los roles, las responsabilidades y las expectativas de cada profesional para un trabajo más completo. Las autoras responden a esa recomendación, en particular al entrecruzar el saber de sus disciplinas. Sin embargo, como toda acción responsable dentro del trabajo clínico, queremos reconocer que otros profesionales en el país realizan trabajo interdisciplinario. La necesidad de documentar este trabajo clínico responde a la responsabilidad de divulgar el quehacer investigativo en beneficio de las disciplinas y las personas involucradas.

El tipo de profesional en el que nos convertimos no es sólo el producto de nuestra formación académica, sino que resulta también 
de las interacciones que mantenemos con quienes nos rodean en el ámbito laboral. Por lo tanto, no se puede asumir que la conducta profesional sea independiente de la historia de las relaciones interpersonales en los contextos laborales. Las relaciones laborales podrían estar enmarcada por la contienda y la lucha de poder por los espacios profesionales o por la cooperación y la solidaridad, y cómo significa esto el marco referencial y la coparticipación entre profesionales para acercarse al sufrimiento. El mismo no puede ser comprendido aisladamente de las relaciones en las que vive una persona ni tampoco al margen de las subjetividades de las propias evaluadoras clínicas. Ambas sentimos una curiosidad intensa por lo que nuestras disciplinas dicen y hacen, y como ese decir facilita o impide una relación vincular entre las disciplinas.

A modo de conclusión, es necesario señalar la complejidad presente en los discursos sobre la discapacidad. No estamos ante un mundo simple con leyes absolutas y modos causalitas o dicotómicos de reaccionar ante situaciones. En vez, construimos vivencias sobre lo que experimentamos. Esta visión de la complejidad demuestra la necesidad de tomar en cuenta las distintas realidades, situaciones, dilemas, entre otros múltiples factores, para comprender la construcción de mundo de la persona y su familia.

Se obliga entonces a asumir un carácter interdisciplinario en el que se reconoce la hipercomplejidad del sujeto, y por ende atiende de manera más amplia las necesidades de éste. Como plantea Morin (2004), el estudio de cualquier aspecto de la experiencia humana ha de ser, por necesidad, multifacético. La complejidad del sujeto no puede ser abordada sólo desde un campo del saber, en tanto somos sujetos complejos, miradas unidireccionales sólo conducen a respuestas simples. El trabajo interdisciplinario permite entonces, un acercamiento a la complejidad desde una mirada amplia y no reduccionista. 


\section{Referencias}

Amores, Sara. (2000). Clínica del niño y su familia: una perspectiva vincular psicoanalítica. Argentina: Editorial Distal.

Bernal, Guillermo \& Rodríguez, Wanda. (1992). Clasificaciones diagnóstica en Puerto Rico: Problemas teóricos, metodológicos y sociopoliticos. Puerto Rico: Publicaciones Puertorriqueñas.

Boyd Webb, Nancy. (Ed.). (2007). Play therapy with children in crisis: A casebook for practitioners ( $3^{\text {rd }}$ ed.). New York: Guilford Press.

Cassidy, Jude \& Shaver, Phillip. (2008). Handbook of attachment: Theory, research, and clinical application $\left(2^{\text {nd }}\right.$ ed.). New York: Guilford Press.

Dell'Anno, Amelia. (2004). El enfoque de la resiliencia como herramienta de trabajo interdisciplinario en el área de la discapacidad. En A. Dell'Anno, M. Corbacho \& M. Serrat (Eds.), Alternativas de la diversidad social: las personas con discapacidad (pp. 123-152). Argentina: Espacio Editorial.

Dolto, Francoise. (1994a). Niño deseado, niño feliz (4 ${ }^{\text {ta }}$ ed.). Argentina: Editorial Paidós.

Dolto, Francoise. (1994b). ¿Tiene el niño derecho a saberlo todo? (4 ${ }^{\text {ta }}$ ed.). Argentina: Editorial Paidós.

Dolto, Francoise. (1998). El niño y la familia: desarrollo emocional y entorno familiar. Argentina: Editorial Paidós.

Fainblum, Alicia. (2004). Discapacidad: una perspectiva clínica desde el psicoanálisis. Argentina: Tekné.

Gil, Eliana. (1994). Play in family therapy. New York: Guilford Press. 
Lejarraga, Horacio y Schejter, Virginia. El trabajo interdisciplinario. En H. Lejarraga (Ed.), (2004). Desarrollo del niño en contexto (pp. 559-571). Argentina: Editorial Paidós.

Linder, Toni. (2008). Transdisciplinary play-based intervention: Guidelines for developing a meaningful curriculum for young children ( $2^{\text {nd }}$ ed.). Florida: Paul H. Brookes Publishing.

López, Félix; Etxebarria, Itziar; Fuentes, María Jesús \& Ortiz, María José. (2000). Desarrollo afectivo y social. España: Ediciones Pirámide.

McLean, Mary; Bailey, Donald \& Wolery, Mark. (2003). Assessing infants and preschoolers with special needs ( $3^{\text {rd }}$ ed.). New Jersey: Prentice Hall

Morin, Edgar. (2004). Introducción al pensamiento complejo. México: Editorial Gedisa.

Pechberty, Bernard. (2002). Lo infantil y la clínica del niño (Trad. Isabel Moreno). España: Editorial Síntesis. (Trabajo original publicado en 2000).

Serrat, Mario. (2004). Sobre los modelos aplicados a la interpretación, clasificación y atención de las discapacidades. En A. Dell'Anno, M. Corbacho \& M. Serrat (Eds.), Alternativas de la diversidad social: las personas con discapacidad (pp.61-114). Argentina: Espacio Editorial. 